\title{
Torsion Dynamic Behaviour of the Ship Hull Made out of Layered Composites
}

\author{
Ionel Chirica \\ "Dunarea de Jos" University of Galati, Domneasca Street, No. 47, 800008 Galati, Romania \\ Correspondence should be addressed to Ionel Chirica; ionel.chirica@ugal.ro
}

Received 23 February 2013; Accepted 6 May 2013

Academic Editor: Irene Penesis

Copyright (C) 2013 Ionel Chirica. This is an open access article distributed under the Creative Commons Attribution License, which permits unrestricted use, distribution, and reproduction in any medium, provided the original work is properly cited.

\begin{abstract}
This paper addresses the dynamic torsion behaviour of the ship hull with very large open decks. A short overview of the past research is presented, and several key findings and behavioral characteristics are discussed. In the paper, the author is focusing on the dynamic part of a new macroelement model used for torsion dynamic analysis of the ship hull made of composite materials. The numerical analysis (using two methods, one of them being a new proposed method) and experiments are developed on a simplified typical hull of a container ship. The torsion analysis is performed on a scale model of a container ship, made of layered composite plates, and the first 5 natural frequencies are determined. The results obtained with the proposed numerical method (software code TORS, made by the author) are compared with the results obtained with FE analysis and with the experiments done on the physical model.
\end{abstract}

\section{Introduction}

In recent years, the improved design, fabrication, and mechanical performance of low-cost composites have led to an increase in the use of composites for large patrol boats, hovercraft, mine hunters, and corvettes. Currently, there are all-composite naval ships up to $80-90 \mathrm{~m}$ long, and this trend continues. It is predicted that from 2020, the hulls for midsized warships, such as frigates that are typically $120-160 \mathrm{~m}$ long, may be built of composite materials [1].

Nowadays, laminated composite panels are gaining popularity in maritime structural applications such as ship hulls, decks, and ship and offshore superstructures. These panels are becoming increasingly used in structural marine applications due to their high specific stiffness and high specific strength [1-4].

Investigations into the vibration and the elastic behaviior of thin-walled structures, especially thin-walled beams with open and closed cross-sections, have been carried out since the early works $[5,6]$.

Monographs written by Kollbrunner and Basler [7], Gjelsvik [8] and a paper written by Kawai and Fujitani [9] are useful references for developing new methods of the thin-walled beam theory and its applications. The intensive research works have been made to develop finite element models that can accurately represent the complex flexuraltorsional warping deformable response of these structures.

Yonghao et al. in [10] present the theory and method of analyzing horizontal-torsion-coupled dynamic behavior of a ship hull with large hatch opening by the transfer matrix method, considering the ship hull as a simplified free-free nonuniform thin-walled beam.

Senjanović and Grubišić in [11] and Senjanovic et al. [12] have developed a flexural and torsion theory of thin-walled girders by introducing the concept of effective stiffness and mass parameters as modal quantities.

In [13], N. I Kim and M. Y. Kim have proposed a general theory for the shear deformable thin-walled beam with nonsymmetric open/closed cross-sections, by developing an improved shear deformable beam theory based on Vlasov's assumption and applying Hellinger-Reissner principle.

In [14], Ascione et al. have developed a one-dimensional kinematical model capable of assessing the dynamic behavior of fibre-reinforced polymers (FRPs) thin-walled beams with open cross-section. The proposed model accounts for the effects of shear deformability.

Vörös, in [15], has proposed a finite element model with seven degrees of freedoms per node. He has also analyzed the free vibration and mode shapes of straight beams. 
The thin-walled beam methodology based code was developed by Chirica et al. [16, 17], Chirica and Beznea, in [18], for static torsion behavior analysis of ship hull with open and closed cross-sections.

The methodology is extended in the present paper to approach the macroelement model for torsion dynamic behavior of the ship hull made of orthotropic layered composite material.

The author considers the methodology presented in the paper to be appropriate and efficient in analyzing torsion vibration problem of the thin-walled laminated composite beam with a special application in ship design activity. The proposed macroelement model offers a facile mode to prepare the input data for the ship hull geometry describing.

\section{Torsion Dynamic Model of the Macroelement}

2.1. General Hypothesis. The methodology presented in the paper is based on a macro element model aimed to analyze the torsion ship hull dynamic treated as thin-walled beam. This methodology proposes an extended model for dynamic analysis using the macro element model treated in [16-19]. In the methodology, the outline of the section is considered as a polygonal one. For a straight line portion of cross-section outline is corresponding a longitudinal strip plate (Figure 1). Due to the torsion loading of the thin-walled beam, in the strip plate the stretching-compression, bending, and shearing occur. The strip plate is treated as an Euler-Bernoulli beam [16-19]. The stiffness matrix and inertia matrix of the macroelement are obtained by assembling the stiffness and inertia matrices of the strips [16].

For macroelement analysis, two coordinate systems are used:

(i) global system $R X Y Z$ having axis $R X$ along the torsion centers line $(R)$ of the cross-sections;

(ii) local system attached to each strip plate $k\left(C_{k} x_{k} y_{k} z_{k}\right)$ having the axis $C_{k} x_{k}$ parallel with $R X$, along the weight centers of the cross-sections of the strip plate.

The macro element is considered to be made of an orthotropic composite material.

The methodology presented in this paper is treating in different ways and different hypothesis, depending on the type of cross-section: open and closed cross-sections in the papers developed in [16-19], where the appropriate mathematical model explaining how the finite strip method is applied for the static analysis of a composite hull is described. Therefore, in this paper, only the dynamic part of the model is presented.

2.2. Composite Characteristics of the Thin Wall. In the next chapter the thin-walled beam theory based methodology is presented, where the ship hull is considered as being made out of isotropic material.

In this paper, the methodology is applied to the thinwalled beam structure of the ship hull made out of orthotropic layered composite plates.

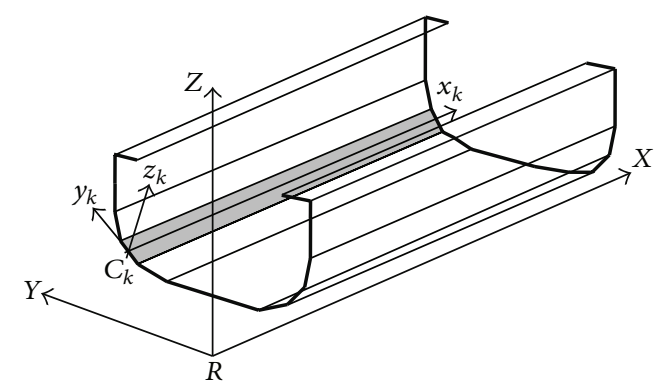

FIgURE 1: The macro-element model and the longitudinal strip.

The equivalent stiffness coefficients for the tensioncompression, bending, shearing, and torsion loading of the strip $k$, having $n s$ layers, have been determined by Chirica et al. in [16-18], according to the condition to have the same stiffnesses for the structure made out in two material cases: isotropic material and orthotropic layered material. This condition is resulting from the equivalent equations of the axial forces $N_{i, k}$, shear forces $F_{i, k}$, and bending moments $M_{i, k}$ acting in the weight centers $\left(C_{i, k}\right)$ of all layers $(i=1, n s)$ belonging to the $k$ th longitudinal strip (see Figure 2 ).

By considering the same geometry for the section (i.e., by keeping the same dimensions $h_{k}$ and $\delta_{k}$ ), the equivalent stiffness coefficients are resulting as follows [16-18]:

(i) for tension-compression

$$
(E A)_{k}=2\left(\sum_{i=1}^{n s} E_{i, k} g_{i, k}\right) h_{k}
$$

(ii) for bending

$$
(E I)_{k}=2 \sum_{i=1}^{n s} E_{i, k} I_{i, k}=\frac{1}{6}\left(\sum_{i=1}^{n s} E_{i, k} g_{i, k}\right) h_{k}^{3} ;
$$

(iii) for torsion

$$
\left(G I_{T}\right)_{k}=8 h_{k} \sum_{i=1}^{n s} G_{i, k} z_{i, k}^{2} g_{i, k}
$$

Also, the equivalent Young's $\left(E_{k}\right)$ and shear $\left(G_{k}\right)$ moduli are obtained as

$$
\begin{gathered}
E_{k}=\frac{2}{\delta_{k}} \sum_{i=1}^{n s} E_{i, k} g_{i, k}, \\
G_{k}=\frac{24}{\delta_{k}^{3}} \sum_{i=1}^{n s} G_{i, k} z_{i, k}^{2} g_{i, k} .
\end{gathered}
$$

In (1)-(4), $E_{i, k}$ and $G_{i, k}$ are the Young's and shear modulus, respectively, of the $i$ th layer, belonging to the $k$ th strip.

The other parameters used in (1)-(4) and Figure 2 are as follows.

(i) $g_{i, k}$ is the thickness of the $i$ th layer belonging to the $k$ th longitudinal strip; 


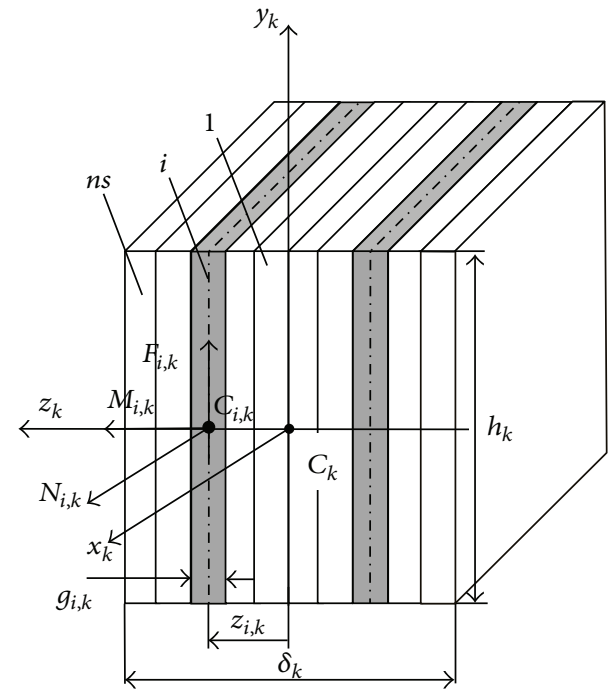

FIGURE 2: Forces acting on the $i$ th layer of the $k$ th longitudinal strip.

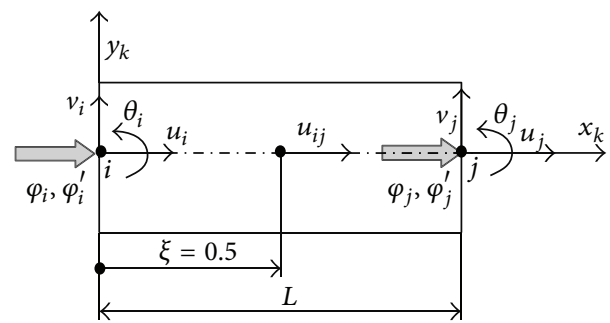

FIGURE 3: Kinematic parameters of the $k$ th longitudinal strip beam.

(ii) $h_{k}$ is the length of the $k$ th longitudinal strip;

(iii) $\delta_{k}$ is the total thickness of the $k$ th longitudinal strip;

(iv) $z_{i, k}$ is the $z$ coordinate (measured along the thickness) of the $i$ th layer belonging to the $k$ th longitudinal strip.

2.3. Specific Matrices of the Macroelement. Initially, to apply the Vlasov's theory, the macroelement is considered to be made of isotropic material, with the characteristics determined in the previous chapter.

For the $k$ th strip's displacements $\varphi(\xi)$ and $v_{k}(\xi)$ (see Figure 3), polynomial functions (third order) are chosen (see [16-18]):

$$
\begin{gathered}
\varphi(\xi)=H_{1}(\xi) \varphi_{i}+L H_{3}(\xi) \varphi_{i}^{\prime}+H_{2}(\xi) \varphi_{j}+L H_{4}(\xi) \varphi_{j}^{\prime} \\
v_{k}(\xi)=H_{1}(\xi) v_{i}^{k}+L H_{3}(\xi) \theta_{i}^{k}+H_{2}(\xi) v_{j}^{k}+L H_{4}(\xi) \theta_{j}^{k} .
\end{gathered}
$$

The polynomial functions used in (5) have the form

$$
\begin{array}{cc}
H_{1}(\xi)=1-3 \xi^{2}+2 \xi^{3}, & H_{2}(\xi)=3 \xi^{2}-2 \xi^{3}, \\
H_{3}(\xi)=\xi-2 \xi^{2}+\xi^{3}, & H_{4}(\xi)=\xi^{3}-\xi^{2},
\end{array}
$$

where $\xi=x / L$.
In (5), $L$ is the strip length (equal to the macro-element length) and

$$
\theta_{i}^{k}=\frac{d v_{i}^{k}}{d x} ; \quad \theta_{j}^{k}=\frac{d v_{j}^{k}}{d x}
$$

The other parameters used in (5) are shown in Figure 3.

For horizontal bending and torsion of the $k$ th longitudinal strip beam, the well-known stiffness and inertia matrices are obtained:

$$
\begin{aligned}
& \mathbf{k}_{v}^{k}=\frac{E I_{k}}{L^{3}}\left(\begin{array}{cccc}
12 & 6 L & -12 & 6 L \\
& 4 L^{2} & -6 L & 2 L^{2} \\
& \text { symm. } & 12 & -6 L \\
& & & 4 L^{2}
\end{array}\right), \\
& \mathbf{k}_{\varphi}^{k}=\frac{G I_{T k}}{L}\left(\begin{array}{cccc}
\frac{6}{5} & \frac{L}{10} & -\frac{6}{5} & \frac{L}{10} \\
& \frac{2 L^{2}}{15} & -\frac{L}{10} & -\frac{L^{2}}{10} \\
& \text { symm. } & \frac{6}{5} & -\frac{L}{10} \\
& & & \frac{2 L^{2}}{15}
\end{array}\right) \text {, } \\
& \mathbf{m}_{v}^{k}=\frac{m L}{420}\left(\begin{array}{cccc}
156 & 22 L & 54 & -13 L \\
& 4 L^{2} & 13 L & -3 L^{2} \\
& \text { symm. } & 156 & -22 L \\
& & & 4 L^{2}
\end{array}\right), \\
& \mathbf{m}_{\varphi}^{k}=\frac{\rho I_{T k} L}{420}\left(\begin{array}{cccc}
156 & 22 L & 54 & -13 L \\
& 4 L^{2} & 13 L & -3 L^{2} \\
& \text { symm. } & 156 & -22 L \\
& & & 4 L^{2}
\end{array}\right),
\end{aligned}
$$

where

(i) $m$ is the distributed mass per unit length;

(ii) $\rho$ is the material density;

(iii) $L$ is the length of macroelement;

(iv) $I_{T k}$ is the conventional polar moment of the inertia of the $k$ th strip plate;

(v) $I_{k}$ is the moment of inertia regarding the lateral bending;

(vi) $E$ and $G$ are the Young's and shear moduli, respectively, of the material.

The equation between stretching-compression and bending degrees of freedom $\left(\mathbf{u}^{k}, \mathbf{v}^{k}\right)$ of the $k$ th strip and degrees of freedom of the macroelement $\varphi$ is obtained as the relation between master and slave nodes:

$$
\mathbf{u}^{k}=\mathbf{C}_{u, \varphi}^{k} \varphi ; \quad \mathbf{v}^{k}=\mathbf{C}_{v, \varphi}^{k} \varphi,
$$


where $\mathbf{C}_{u, \varphi}^{k}$ and $\mathbf{C}_{v, \varphi}^{k}$ are "form" matrices of the strip $k$, presented in [16-18]. The displacements vectors are

$$
\mathbf{u}^{k}=\left(\begin{array}{c}
u_{i}^{k} \\
u_{i j}^{k} \\
u_{j}^{k}
\end{array}\right) ; \quad \varphi=\left(\begin{array}{c}
\varphi_{i} \\
\varphi_{i}^{\prime} \\
\varphi_{j} \\
\varphi_{j}^{\prime}
\end{array}\right)
$$

Based on the coupling matrices for each strip beam, the strips' stiffness and inertia matrices for axial and bending loading are assembling to obtain the stiffness and inertia matrices of the macroelement. The assembling process is performed by the transforming process using the orthogonal process [20].

The stiffness matrix of the macroelement is obtained by

$$
\mathbf{K}=\frac{E I_{\omega}}{L^{3}} \widetilde{\mathbf{K}}_{E B}+\frac{G I_{T}}{L} \widetilde{\mathbf{K}}_{S V}
$$

In (11), the matrices $\widetilde{\mathbf{K}}_{E B}$ and $\widetilde{\mathbf{K}}_{S V}$ are stiffness matrix for warping torsion and stiffness matrix for free torsion, developed in [16-19].

The inertia matrix of the macroelement is obtained by

$$
\mathbf{M}=\frac{\rho I_{\omega}}{L} \widetilde{\mathbf{M}}_{E B}+\rho I_{T} L \widetilde{\mathbf{M}}_{S V}
$$

In (12), the matrices $\widetilde{\mathbf{M}}_{E B}$ and $\widetilde{\mathbf{M}}_{S V}$ have the forms

$$
\begin{gathered}
\widetilde{\mathbf{M}}_{E B}=\frac{1}{30}\left(\begin{array}{cccc}
36 & 3 L & -36 & 6 L \\
& 4 L^{2} & -3 L & \frac{L^{2}}{2} \\
& \text { symm. } & 36 & -6 L \\
& & & L^{2}
\end{array}\right) ; \\
\widetilde{\mathbf{M}}_{S V}=\frac{1}{420}\left(\begin{array}{cccc}
156 & 22 L & 54 & -13 L \\
& 4 L^{2} & 13 L & -3 L^{2} \\
& \text { symm. } & 156 & -22 L \\
& & & 4 L^{2}
\end{array}\right) .
\end{gathered}
$$

After the coupling of all macro-elements, the inertia matrix $(\overline{\mathbf{M}})$ and stiffness matrix $(\overline{\mathbf{K}})$ of the ship hull are obtained. The equation of natural vibrations of the ship hull has the form

$$
\overline{\mathbf{M}} \ddot{\Delta}+\overline{\mathbf{K}} \Delta=\mathbf{0},
$$

where $\ddot{\Delta}$ and $\Delta$ are the accelerations and displacements vectors, respectively.

Assuming that $\Delta=\Delta_{0} e^{i p t}$, where $\Delta_{0}$ and $p$ are the mode vector and natural frequency, respectively, (14) results in the eigenvalue problem

$$
\left(\overline{\mathbf{K}}-p^{2} \overline{\mathbf{M}}\right) \Delta_{0}=\mathbf{0}
$$

\section{Numerical Analysis: FEM Code TORS versus FE Analysis}

Based on the methodology described in the previous chapter, a macroelement-based code was developed. The code TORS, developed by the author for static analysis in [16-18] and now extended for dynamic numerical analysis, may be applied to ship hull behavior loaded to torsion. For the present study, a simplified model of a container ship, made of composite materials, was analyzed. This type of ship has a very large deck opening for an efficient maneuvering of the containers during loading-unloading process. Therefore, in the middle part of the ship, the deck is reduced to two longitudinal strips, placed on both sides (see Figure 8).

The main characteristics of the model are length $L_{m}=$ $2.4 \mathrm{~m}$, breadth $B_{m}=0.4 \mathrm{~m}$, and depth $D_{m}=0.2 \mathrm{~m}$. The length of the open area is $1.2 \mathrm{~m}$, and the lengths of the closed areas are $0.6 \mathrm{~m}$. The material is E-glass/polyester having the characteristics determined by experimental tests in $[21,22]$ :

$$
\begin{aligned}
& E_{x}=46 \mathrm{GPa}, E_{y}=46 \mathrm{GPa}, E_{z}=13 \mathrm{GPa}, G_{x y}=5 \mathrm{GPa}, \\
& G_{x z}=5 \mathrm{GPa}, G_{y z}=4.6 \mathrm{GPa}, \rho=2045 \mathrm{~kg} / \mathrm{m}^{3}, \\
& \mu_{x y}=0.3, \mu_{y z}=0.42, \mu_{x z}=0.3 .
\end{aligned}
$$

The ship hull model is divided into two parts: the aft part and fore part with closed cross-sections, and the middle part, having open cross-sections (see Figure 8).

The thicknesses' values of the hull shell are $2 \mathrm{~mm}$ for side shell and $3 \mathrm{~mm}$ for deck and bulkheads. The model is a simplified ship hull of a container ship, having the prismatic shell.

To fulfill the thin-walled beam hypothesis (nondeformability of the cross section), the model has 13 transversal bulkheads placed at every $0.2 \mathrm{~m}$ (see Figure 8 ). The influence of the bulkheads on the ship hull torsion behavior was considered by introducing the supplementary inertias on the lateral displacement and torsion angle on the macro-element level.

The first numerical model is the 3D model using macroelements from TORS code.

In the beginning, a convergence analysis was performed. Finally, the optimum number of strip elements and the optimum lengths of the macroelements were determined.

Therefore, model mesh used for TORS calculus is concerning 12 macro-elements: 6 macro-elements in the closed parts ( 3 macro-elements for each end part) of the ship model and 6 macro-elements in the open part (middle part). Each macro-element, representing a piece of ship model, was modeled with longitudinal plane strip elements. The closed section type macro-elements concern 14 strip elements (Figure 4). The open section type macro-elements are modeled with 12 longitudinal strip elements as it is seen in Figure 5. The total number of strip elements (having the same length of $0.2 \mathrm{~m}$ ) is 228 . The outline of the cross section of the model was approached with a polygonal line. The bilge was modelled with 3 longitudinal strip elements (S2, S3, and S4 in Figures 4 and 5).

The second numerical model is a 3-D model with 4-node shell composite finite elements. 


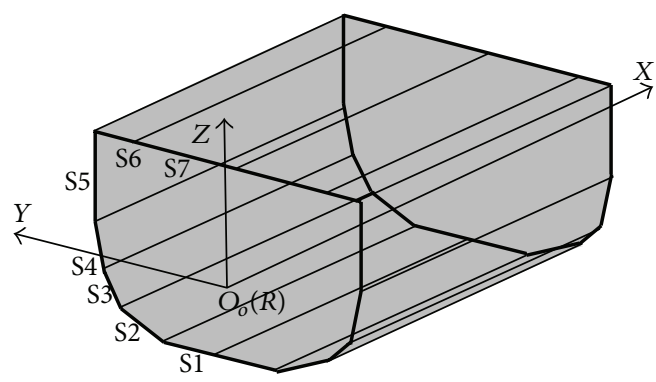

FIGURE 4: The mesh concerning longitudinal strips for closed section.

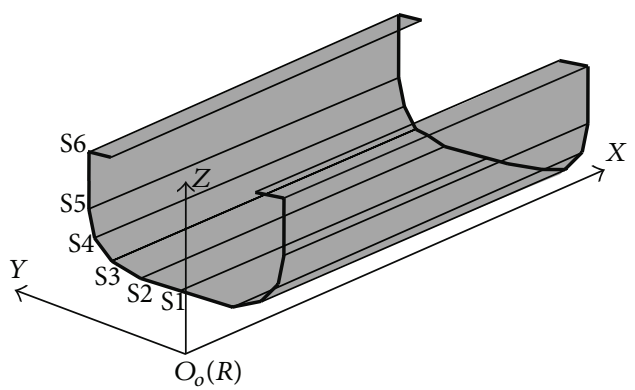

FIGURE 5: The mesh concerning longitudinal strips for open section.

In the beginning, a convergence analysis was performed [18]. Finally, the optimum dimension of the quadrilateral element side $(0.02 \mathrm{~m})$ was determined, and a number of 18110 shell elements were used in the mesh model.

The results obtained with the code TORS were compared with the results obtained by FE analysis (see Table 1). Due to the fact that the real ship has much stiffened structure in both ends, in the both modeling types (TORS and FE analysis) the model is considered as clamped at the ends.

The graphical representations of the first 3 vibration modes in air, presented in the Figure 6, give a good information about the differences in torsion stiffness between closed and opened section (for the ship hull model the torsion stiffnesses ratio is about $10^{4}$ ).

On this count, the numerical analysis of the simplified model (named as "full" model) was further done by considering only the open part of the model (named as "open" model), clamped in the joining sections between opened and closed parts. The results obtained after numerical calculus with TORS are presented in Figure 7 as a graphical zoom of the first torsion modal deformed shape (variation of torsion angle) in the area between sections placed at $0.4 \mathrm{~m}$ and $0.6 \mathrm{~m}$. The curve 1 represents variation of torsion angle for "full" model. The curve 2 represents variation of torsion angle for "open" model. As it is seen, the both curves are approaching in the open area. The results obtained for the first 5 frequencies of both models (model 1 is considered as "full" model and model 2 is considered as "open" model) are presented in the Table 1.
TABLE 1: Natural frequencies $(\mathrm{Hz})$.

\begin{tabular}{lccccc}
\hline $\begin{array}{l}\text { Mode } \\
\text { no. }\end{array}$ & $\begin{array}{c}\text { TORS } \\
\text { Model 1 }\end{array}$ & $\begin{array}{c}\text { FE } \\
\text { Model 1 }\end{array}$ & $\begin{array}{c}\text { TORS } \\
\text { Model 2 }\end{array}$ & $\begin{array}{c}\text { FE } \\
\text { Model 2 }\end{array}$ & Experiment \\
\hline 1 & 309 & 301 & 300 & 298 & 290 \\
2 & 329 & 319 & 323 & 313 & 307 \\
3 & 353 & 342 & 347 & 337 & 332 \\
4 & 368 & 373 & 361 & 367 & 359 \\
5 & 416 & 411 & 403 & 406 & 395 \\
\hline
\end{tabular}
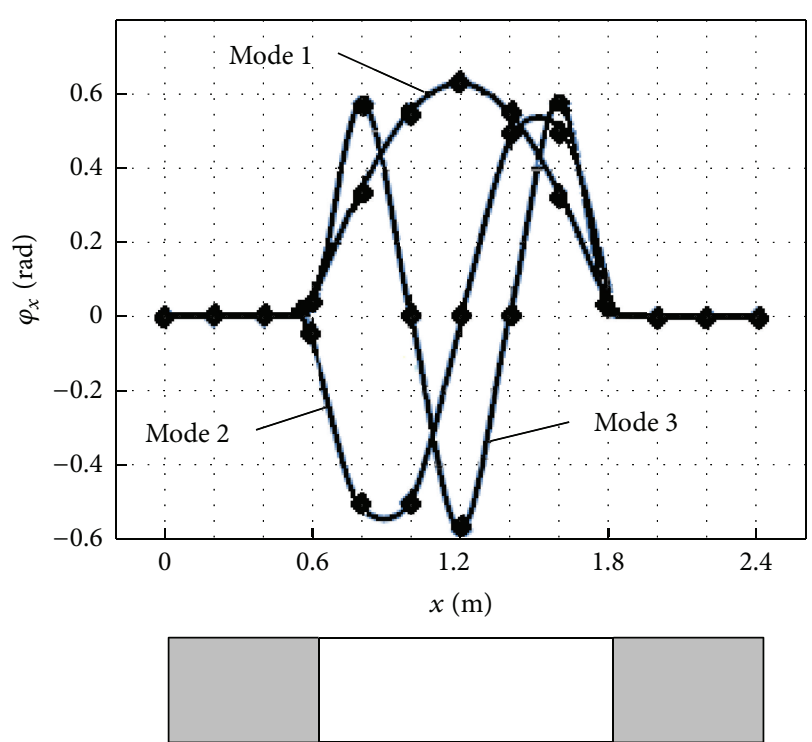

FIGURE 6: The first three vibration modes in air, obtained by numerical calculus with TORS.

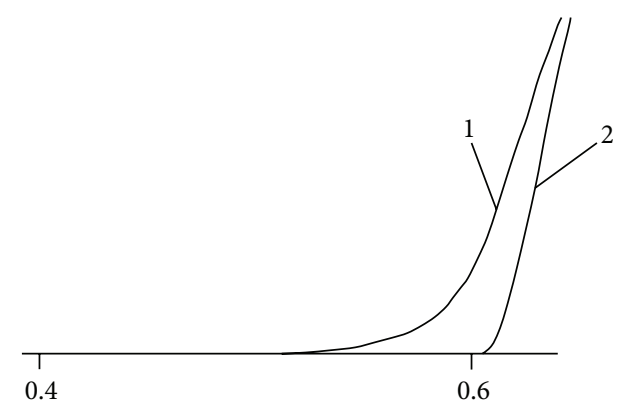

FIGURE 7: Graphical zoom for vibration modes.

\section{Experimental Tests}

It is often difficult to achieve frequencies and mode shapes with complex simulation models used in a model test. Numerical model tested with TORS was verified with a demonstrator of a simple ship hull model made out of layered composites.

The test rig for torsional vibrations estimation of the ship hull model is shown in Figure 8. The torque is obtained with a dynamic couple of two forces acting on a frame placed in the midship section of the ship model. 


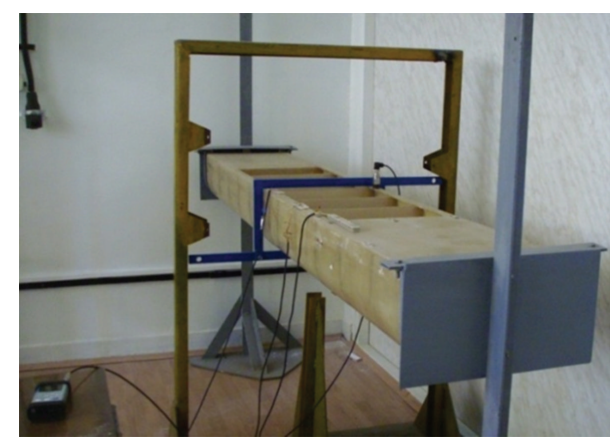

(a)

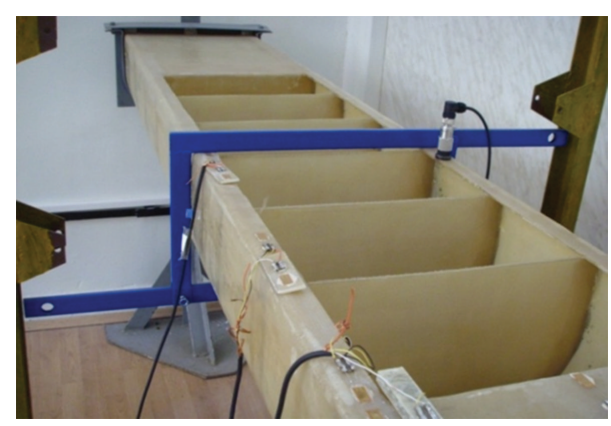

(b)

Figure 8: Test rig.

The natural frequencies of the model were determined with an equipment concerning a vibrometer and an accelerometer. The second measuring equipment used for experiments consists of gauges measurements. Both equipments have generated the results in a good agreement.

Impact excitation method is used in this study to predict the natural frequencies of the model. The impact excitations were obtained with a special hammer.

By striking the model at any point on the special frame placed on the middle section of the model and displaying the response signals from the accelerometer in the frequency domain, the fundamental natural frequencies were measured at resonant peaks. To determine the torsion-horizontal bending mode shape corresponding to the natural frequency of the model, a large number of repeated tests were done (see Figure 8). To obtain the best results for torsion coupled with horizontal bending vibrations, the accelerometer was placed in various positions on deck plate into side part of the model. The vertical-bending natural frequencies were filtered according to the calculations done for this type of vibrations.

The aim of the work was to determine the frequencies of the ship hull (global vibrations). To avoid the detecting of the local vibrations of the panels (not interested in this work), the accelerometer was placed only in the correspondence of the transversal bulkheads, or very close to the special frame.

Data collection was performed according to a test program and procedure:

(i) the determining of the appropriate measuring points (positions of the accelerometers and strain gauges), chosen so that to avoid as much as possible of the local vibrations;

(ii) the determining of the appropriate impact points (positions of the hammer) on the frame so that to perform as much as possible of only global vibrations exciting (and not the frame vibrations);

(iii) collecting data with accelerometers and strain gauges during impact excitations;

(iv) data processing;

(v) post-test verification. The model test was verified by post-test analysis. This analysis was feeding the measured natural frequencies back into the numerical model of the ship hull model by comparing the numerical and experimental frequencies. The purpose of this check is to verify that the global conditions imposed in the numerical simulation (boundary conditions) of the model provide a good agreement with the global conditions of the experimental model;

(vi) the boundary conditions were reconsidered into the numerical analysis, and the experimental testings were remade.

The fundamental natural frequencies of the model can be predicted from the frequency domain of the accelerometer response. The first 5 frequencies obtained from experiments give the values closed to the natural frequencies obtained from numerical analysis (TORS and FE analysis) as are shown in the Table 1.

\section{Conclusions}

The proposed methodology is a developed and extended theory from static to dynamic problems, presented in [16-18].

The methodology was found to be appropriate and efficient in analyzing torsion vibration problem of a thinwalled laminated composite beam with a special application in ship design activity.

The facile mode to prepare the input data for defining the cross sections and finally the entire ship hull, by using macroelement model and code TORS, recommends the methodology as a good tool for torsion analysis of ship hull made of composite materials. Additionally, the CPU time machine with TORS is much lesser than the CPU time obtained with FE analysis [18].

Comparison between the results obtained in numerical and experimental analysis shows good agreements for the resonance frequencies in most cases.

Also, the close comparation between FEA analysis and TORS calculus provides close values for the natural frequencies and modal shapes and reveals macro-element modelbased methodology as a good and efficient tool. 
Certain remarks can be developed.

(i) The analysed model is a prismatic one, to avoid the distortions in results due to sudden variation of ship forms on the ends.

(ii) As it is seen in the graphical representations in Figure 6 due to very huge torsion stiffness difference between closed and opened sections, the ship hull ends may be considered as clamped.

(iii) According to the torsion deformations modes, the approach calculations can be done only for the open part of the ship, considering the middle part as being clamped in the joint sections with closed parts. This approach calculus can be done with fair results for the design stage of the ship hulls with large deck openings.

(iv) Due to the variation of the cross section shape of the model, a coupled torsion vibrations with lateral bending vibrations that cannot be separated occurred. Therefore, the frequencies for each of both vibration types cannot be separated.

(v) The results obtained by the three methods (macroelement model-TORS software, FE analysis, and experimental tests) are in a fair agreement.

\section{Acknowledgment}

The work has been performed in the scope of the National Project 168 EU-UEFISCDI (2012-2014).

\section{References}

[1] A. P. Mouritz, E. Gellert, P. Burchill, and K. Challis, "Review of advanced composite structures for naval ships and submarines," Composite Structures, vol. 53, no. 1, pp. 21-24, 2001.

[2] I. Chirica and E. F. Beznea, "Buckling analysis of the composite plates with delaminations," Computational Materials Science, vol. 50, no. 5, pp. 1587-1591, 2011.

[3] I. Chirica, D. Boazu, and E. F. Beznea, "Response of ship hull laminated plates to close proximity blast loads," Computational Materials Science, vol. 52, no. 1, pp. 197-203, 2012.

[4] E. F. Beznea and I. Chirica, "Buckling behaviour of imperfect composite plate," Revista de Materiale Plastice, vol. 48, no. 3, pp. 231-239, 2011

[5] V. Z. Vlasov, Thin-Walled Elastic Beams, Program of Scientific Translations, Jerusalem, Israel, 2nd edition, 1961.

[6] S. P. Timoshenko and J. M. Gere, Theory of Elastic Stability, McGraw-Hill, New York, NY, USA, 1961.

[7] C. F. Kollbrunner and K. Basler, Torsion in Structures, Springer, Berlin, Germany, 1969.

[8] A. Gjelsvik, The Theory of Thin Walled Bars, John Wiley \& Sons, New York, NY, USA, 1981.

[9] T. Kawai and Y. Fujitani, Some Considerations on the Modern Beam Theory-Development of the Practical Methods, vol. 32 of Report of the Institute of Industrial Science, The University of Tokyo, 1986.

[10] P. Yonghao, P. Yougang, and Y. Xueyong, “The horizontaltorsion-coupled model analysis and dynamic response calculation of the ship hull with a large hatch opening," Journal of Tianjin University, pp. 51-58, 1993.
[11] I. Senjanović and R. Grubišić, "Coupled horizontal and torsional vibration of a ship hull with large hatch openings," Computers and Structures, vol. 41, no. 2, pp. 213-226, 1991.

[12] I. Senjanovic, S. Tomasevic, and R. Grubisic, "Coupled horizontal and torsional vibrations of container ships," Brodogradnia, vol. 58, no. 4, pp. 365-379, 2007.

[13] N. I. Kim and M. Y. Kim, "Exact dynamic/static stiffness matrices of non-symmetric thin-walled beams considering coupled shear deformation effects," Thin-Walled Structures, vol. 43, no. 5, pp. 701-734, 2005.

[14] L. Ascione, L. Feo, and G. Mancusi, "On the statical behaviour of fibre-reinforced polymer thin-walled beams," Composites $B$, vol. 31, no. 8, pp. 643-654, 2000.

[15] G. M. Vörös, "Free vibration of thin-walled beams," Journal of Computational and Applied Mechanics, vol. 9, no. 2, pp. 1-14, 2008.

[16] I. Chirica, S. D. Musat, R. Chirica, and E. F. Beznea, “Torsional behaviour of the ship hull composite model," Computational Materials Science, vol. 50, no. 4, pp. 1381-1386, 2011.

[17] I. Chirica, D. Boazu, E. F. Beznea, and A. Chirica, "Ship hull composite plates analysis under blast loads," in Proceedings of the of the 3rd International Conference on Advances in Marine Structures (MARSTRUCT '11), pp. 343-350, Taylor \& Francis, Hamburg, Germany, 2011.

[18] I. Chirica and E. F. Beznea, "A numerical model for torsion analysis of composite ship hulls," Mathematical Problems in Engineering, vol. 2012, Article ID 212346, 17 pages, 2012.

[19] I. Chirica, E. F. Beznea, and D. Boazu, "Torsion dynamic analysis of a ship hull composite model," in Proceedings of the of the 14th International Congress of International Maritime Association of the Mediterranean, vol. 1, pp. 331-337, Taylor \& Francis, Genova, Italy, 2011.

[20] S. K. Ider and F. M. L. Amirouche, "Coordinate reduction in the dynamics of constrained multibody systems-a new approach," Journal of Applied Mechanics, vol. 55, no. 4, pp. 899-904, 1988.

[21] E. F. Beznea, Studies and researches on the buckling behaviour of the composite panels [Doctoral thesis], University Dunarea de Jos of Galati, 2008.

[22] E. F. Beznea, Composite Structures-Laboratory Applications, Galati University Press, 2012 (Romanian). 


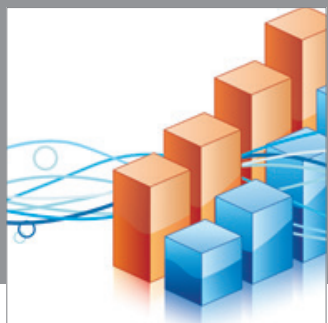

Advances in

Operations Research

mansans

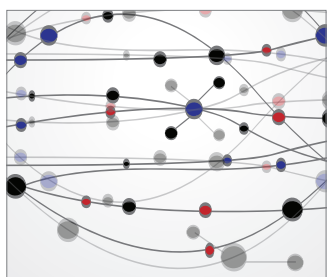

The Scientific World Journal
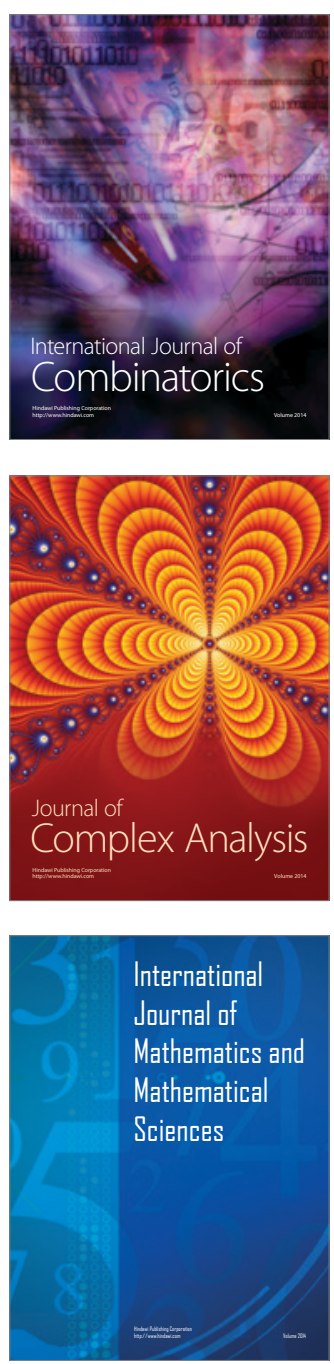
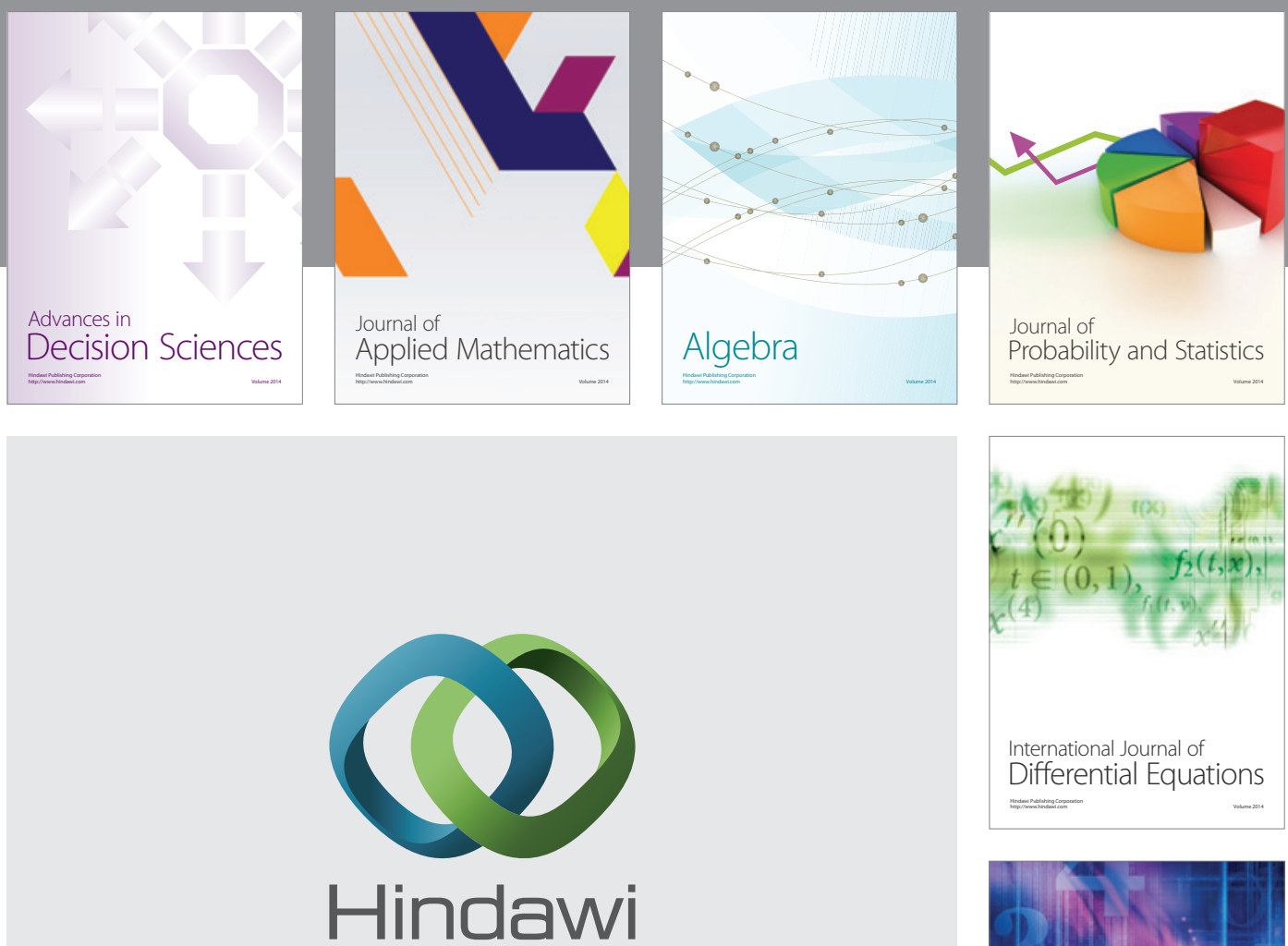

Submit your manuscripts at http://www.hindawi.com
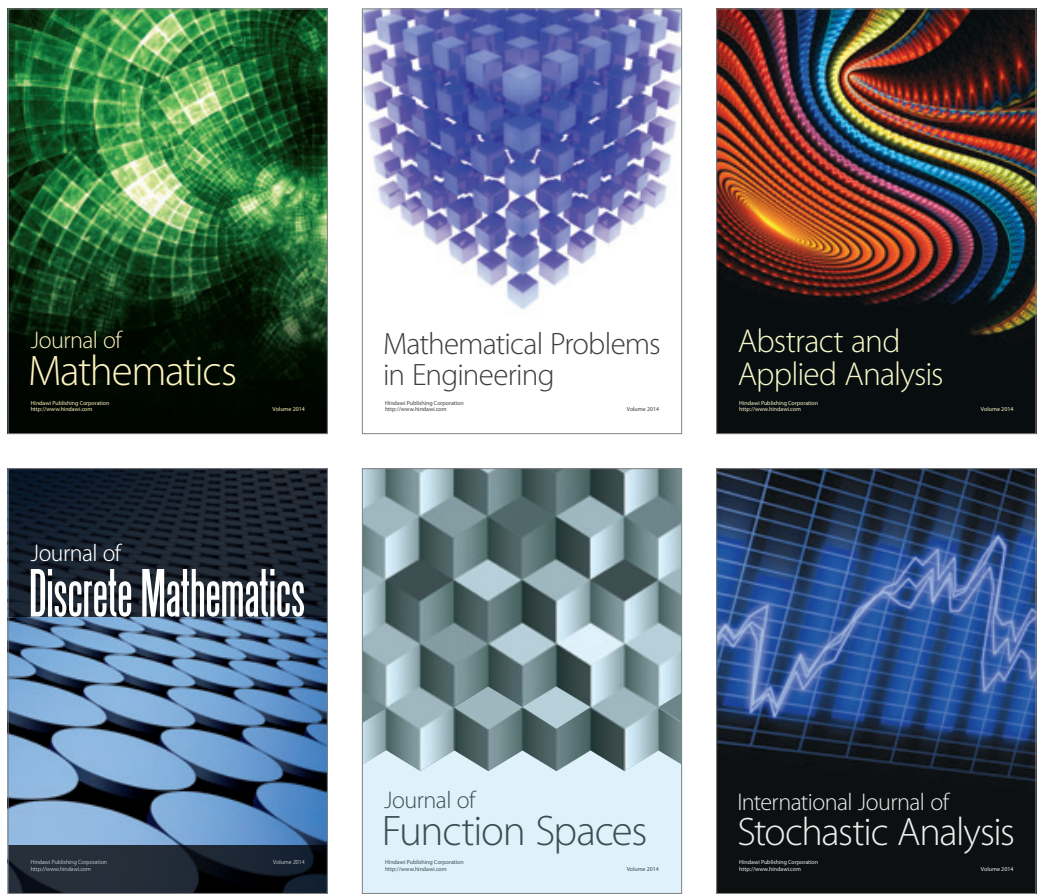

Journal of

Function Spaces

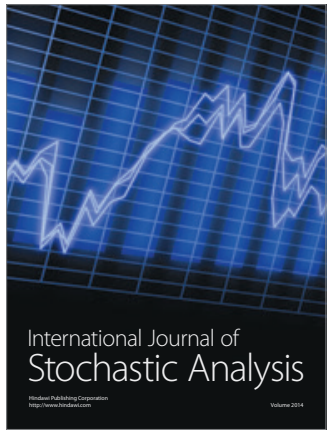

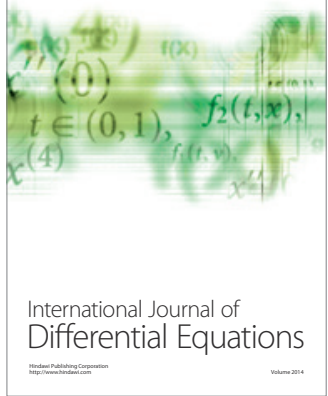
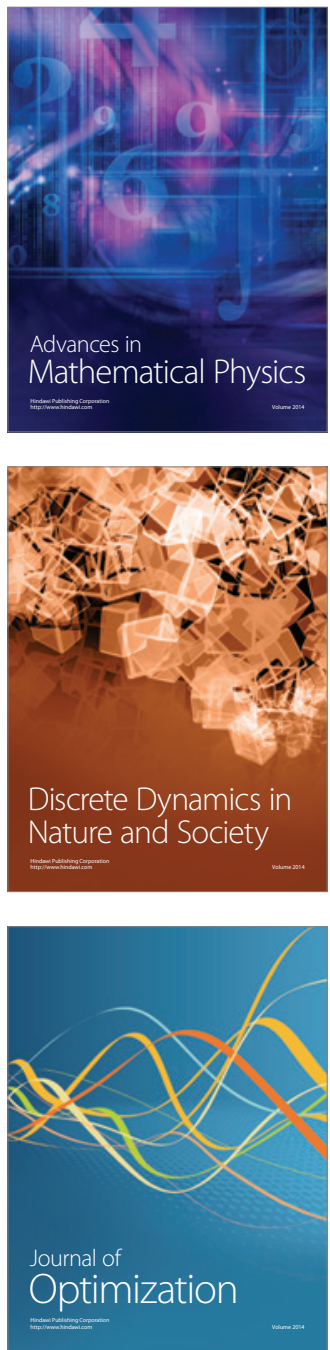